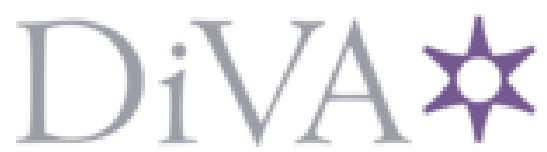

http://www.diva-portal.org

Preprint

This is the submitted version of a paper presented at 2018 IEEE International Symposium on Information Theory, ISIT 2018, Vail, United States, 17 June 2018 through 22 June 2018.

Citation for the original published paper:

Vu, M T., Oechtering, T J., Skoglund, M., Boche, H. (2018)

Uncertainty in Identification Systems

In: IEEE International Symposium on Information Theory - Proceedings, 8437760

(pp. 2386-2390). Institute of Electrical and Electronics Engineers (IEEE)

IEEE International Symposium on Information Theory - Proceedings

https://doi.org/10.1109/ISIT.2018.8437760

N.B. When citing this work, cite the original published paper.

The work was supported by the Swedish Research Council (VR) project under Grant 2016-03853.

Permanent link to this version:

http://urn.kb.se/resolve?urn=urn:nbn:se:kth:diva-234484 


\title{
Uncertainty in Identification Systems
}

\author{
Minh Thanh Vu*, Tobias J. Oechtering*, Mikael Skoglund* and Holger Boche ${ }^{\dagger}$ \\ * Dept. ISE, KTH Royal Institute of Technology \\ $\dagger$ LTI, Technische Universität München
}

\begin{abstract}
We study the high-dimensional identification systems under the presence of statistical uncertainties. The task is to design mappings for enrollment and identification purposes. The identification mapping compresses users' information then stores the index in the corresponding position in a database. The identification mapping combines the information in the database and the observation which originates randomly from an enrolled user to produce an estimate of the underlying user index. We study two scenarios. Users' data are generated from the same unknown distribution while the observation channel is also subjected to uncertainty. Each user's data are generated iid from the distribution corresponding to its own state, while the observation channel is known. We provide an achievable compression-identification trade-off for the first and second settings considering both discrete and continuous cases. In the discrete scenario, the described regions are also the correspondingly complete characterizations.
\end{abstract}

\section{INTRODUCTION}

Consider a forensic system where biometric information such as facial features, height, fingerprint etc. are taken and stored into a database. Compression of this information is desirable to reduce storage burdens. The system is required to work properly regardless of users' (ethnic) background information. Additionally, the robustness requires the identification of the true user with high probability when an observation, which undergoes an uncertain random transformation, is provided to the processing unit.

Willems et.al [1] characterized the capacity of a biometric identification system with noisy data and observation sequences. The study has been developed further in several directions. In [2] and [3] the compression of users' information and distortion aspects were taken into account. Therein, the authors characterized the trade-off between the compressionidentification rate (compression-identification-distortion tradeoff) where the both the distribution of users' data and the observation channel are known. The compression of observation under the known statistics was considered in [4].

In another line of work, the channel capacity for a compound and an arbitrarily varying setting were first established in [5], [6]. Ahlswede showed in [7] that the capacity under the average error constraint is either zero or equal to the random code capacity. A more complete account for the study of arbitrarily varying channel is given in [8, Chapter 12].

Motivated from the above example, in this work we study identification settings which involve some uncertainties in the distribution of users' data and observation channel. Specifically, we first consider a setting where users' data are generated iid from the same but unknown distribution taken from a finite set. Additionally, we assume that the observation channel is chosen from another finite set of conditional distributions. This setting fits to the scenario that the users belong to the same group and the system is required to work irrespective of the underlying group.

Next, we extend our consideration to the case where the uncertainty varies arbitrarily. We consider a case where users' underlying states are chosen independently from a distribution unknown to us, i.e., users' states are not necessarily identical. Each user's data are then generated iid from the distribution corresponding to its given state. We assume in this case the observation channel is fixed. This setting represents a scenario where the group of users in the system is a mixture from different background groups.

In the first setting, we provide an achievable compressionidentification rate region for both the discrete case and the continuous case. The achievability is proven by a combination of classifiers and typical random coding arguments. The classifiers, which are based on the concentration property of probability measures at large block length, are used to estimate the underlying states for each user's data sequence and observation channel. Moreover, in the discrete setup we provide a converse result that completes the compressionidentification rate region.

Interestingly, in the discrete case the converse result of the setting with independent users' underlying states shows that the compression-identification rate region is identical to the one from the first setting. In the continuous case, the corresponding region from the first setting is also achievable. The achievability in both cases: discrete and continuous employs the same technique from the first setting.

To gain a better understanding of the results of the previous settings, we establish a connection between the identification setting and the Wyner-Ahlswede-Körner network [9] and [10]. We show that an identification scheme can be built from a WAK-code which satisfies the desired property. This observation is extended to our first and second settings to show that these two share a class of optimal mappings due to the vanishing estimation errors and the independence conditioning on underlying states.

The paper is organized as follows. In Section II we study the first setting. The study of independent states and the connection to WAK-network is given in Section III.

\section{IDENTIFICATION PROBLEM: COMPOUND SETTING}

\section{A. Problem statement and result}

We first recap the generic setup for an identification system. Data sequences $\left(x^{n}(i)\right)_{i \in \mathcal{W}}$ from $M$ users, where $M=|\mathcal{W}|$, 


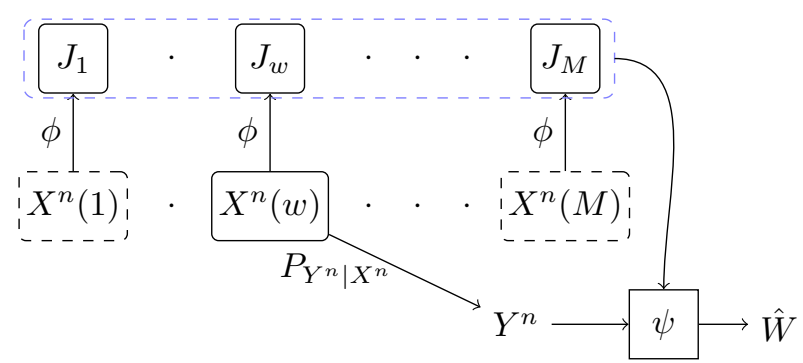

Fig. 1. The generic model for the high-dimension identification system.

are enrolled and stored as $\left(j_{i}\right)_{i=1}^{M}$ in a database. An observation $y^{n}$ which is correlated with a sequence $x^{n}(w)$ where $w$ is chosen uniformly from $\mathcal{W}$ is provided to the processing unit. The processing unit identifies the true user $w$ by using $y^{n}$ and $\left(j_{i}\right)_{i=1}^{M}$. The setup is depicted in Fig. 1 .

In this section we consider the setting where users' data are generated iid from an unknown distribution that belongs to a set. Assume that $P_{X}$ is a member of

$$
\mathcal{P}=\left\{P_{X \mid S=s} \mid s \in \mathcal{S}\right\},
$$

which is a set of probability measures on the same measurable space $(\mathcal{X}, \mathcal{F})$. We can think that nature selects $s \in \mathcal{S}$ which we do not know. Given $S=s$ the corresponding users' data sequences $\left(x^{n}(i)\right)_{i=1}^{M}$ are generated from $P_{X \mid S=s}^{\otimes n}$. Furthermore, the observational channel is from the set

$$
\mathcal{P}_{c}=\left\{P_{Y \mid X, \tau} \mid \tau \in \mathcal{T}\right\},
$$

where $P_{Y \mid X, \tau}$ is a shorthand notation for $P_{Y \mid X, T=\tau}$. Throughout this setting both $\mathcal{S}$ and $\mathcal{T}$ are finite. Denote the corresponding set of "marginal" distributions on $\mathcal{Y}$ by

$$
\mathcal{P}_{\mathcal{Y}}=\left\{P_{Y} \mid P_{Y}=P_{Y \mid X, \tau} P_{X \mid S=s}, \text { for some }(\tau, s)\right\},
$$

i.e., we allow different combinations of channels and input distributions resulting in the same (marginal) output distribution however no input distribution results in the same output distribution with different channels. We enumerate elements of $\mathcal{P}_{\mathcal{Y}}$ by $P_{Y, \kappa}$ where $\kappa \in\left[1:\left|\mathcal{P}_{\mathcal{Y}}\right|\right]$.

Definition 1. A identification scheme consists of an enrollment mapping

$$
\phi: \mathcal{X}^{n} \rightarrow \mathcal{M}_{1}
$$

which compresses users' information and stores it in a database, and an identification mapping $\psi$

$$
\psi: \mathcal{Y}^{n} \times \mathcal{M}_{1}^{M} \rightarrow \mathcal{W} \cup\{e\},
$$

which identifies the true user from the observation and the stored information in the database ${ }^{1}$. Note that both mappings are deterministic.

Definition 2. A compression-identification rate pair $\left(R_{c}, R_{i}\right)$ is achievable if for every $\delta>0$ there exists a pair of aforementioned mappings $(\phi, \psi)$ such that

$$
\frac{1}{n} \log \left|\mathcal{M}_{1}\right|<R_{c}+\delta, \quad \frac{1}{n} \log |\mathcal{W}|>R_{i}-\delta
$$

\footnotetext{
${ }^{1}$ These mappings are Borel measurable.
}

$$
\sup _{\tau, s} \operatorname{Pr}\{W \neq \hat{W} \mid S=s, T=\tau\} \leq \delta .
$$

for all $n \geq n_{0}(\delta)$. The set of all achievable rate pair is denoted by $\mathcal{R}_{1}$.

Definition 3. If $X$ and $Y$ are discrete random variables which take values on finite alphabets $\mathcal{X}$ and $\mathcal{Y}$, we define $\overline{\mathcal{R}}_{1}$ to be the union over the set of distribution $\left\{P_{U \mid X, S=s}\right\}_{s}$ on $|\mathcal{U}| \leq$ $|\mathcal{X}|+|\mathcal{T}|$ of the rate pairs $\left(R_{c}, R_{i}\right)$ for which we have

$$
\begin{aligned}
& R_{c} \geq \max _{s, \tau} I(X ; U \mid S=s, T=\tau) \\
& R_{i} \leq \min _{s, \tau} I(Y ; U \mid S=s, T=\tau) .
\end{aligned}
$$

Otherwise, if $X$ and $Y$ are continuous random variables where $\mathcal{P}$ can be represented by a set of density functions $p_{X \mid S=s}$ and $\mathcal{P}_{c}$ can be represented by a set of conditional density functions $p_{Y \mid X, \tau}$ then $\overline{\mathcal{R}}_{1}$ is defined similarly as the closure of (7) over the set of test channels ${ }^{2} p_{U \mid X, S=s}$.

Theorem 1. For given sets $\mathcal{P}$ and $\mathcal{P}_{c}$, the region $\overline{\mathcal{R}}_{1}$ is achievable, i.e.,

$$
\overline{\mathcal{R}}_{1} \subseteq \mathcal{R}_{1} .
$$

Furthermore, if further $X$ and $Y$ are discrete then we have

$$
\mathcal{R}_{1}=\overline{\mathcal{R}}_{1} .
$$

Due to the uncertainty caused by the underlying states of the users' data and the observation channel, it is intuitive to estimate these parameters. The estimation can be done due to the concentration effects at large block length. In the proof of Theorem 1, we use the two following supporting lemmas. Lemma 1 says that as long as elements in the set of the users' data distributions $\mathcal{P}$ are well-separated then with high probability we can estimate its underlying state correctly.

Lemma 1. Assume that the alphabet $\mathcal{X}$ is a Polish space, specifically, a finite set with discrete metric or $\mathbb{R}$ with Euclidean distance, and $\mathcal{F}$ is the corresponding $\sigma$-algebra generated by open sets. Assume the distributions $P_{X \mid S=s}$ are distinct for all $s \in \mathcal{S}$, i.e., $\forall s \in \mathcal{S}$ we have $d\left(P_{X \mid S=s}, P_{X \mid S=s^{\prime}}\right) \neq 0$ where $d$ is any metric on the space of probability measures. Then there exists a classifier $T: \mathcal{X}^{n} \rightarrow \mathcal{S} \cup\{e\}$, where $e$ denotes an error, such that if $X^{n} \sim P_{X \mid S=s}^{\otimes n}$ then

$$
\operatorname{Pr}\left(T\left(X^{n}\right)=s \mid S=s\right) \rightarrow 1 \text {, as } n \rightarrow \infty .
$$

The proof of Lemma 1 is given in [11, Appendix A]. Since the channel state is also unknown, we can also use Lemma 1 to estimate the unknown parameter $\kappa$. Then, if we can estimate the state $s$ of the true user correctly then by using $\kappa$ and $s$ we know for sure the value of $\tau$. Since all users in the system have the same underlying state, Lemma 2 states that we can use a voting mechanism that decides for the correct state with high probability.

Lemma 2. Assume that $X^{n}(i) \sim P_{X \mid S=s}^{\otimes n}$ for all $i \in[1$ : $M]$, where $s \in \mathcal{S}$ is fixed for all indices $i$. Let $T_{X}$ be the

\footnotetext{
${ }^{2}$ We require that the entropy and mutual information are finite.
} 
classifier as in Lemma 1 and denote $\hat{S}_{i}=T_{X}\left(X^{n}(i)\right)$. Let ${ }^{3}$ $S^{\star}=\arg \max _{\hat{s} \in \mathcal{S} \cup\{e\}} \frac{1}{M} \sum_{i=1}^{M} \chi_{\left\{\hat{S}_{i}=\hat{s}\right\}}$. Then

$$
\operatorname{Pr}\left\{S^{\star}=s \mid S=s\right\} \rightarrow 1
$$

as $n \rightarrow \infty$.

The proof of Lemma 2 is given in [11, Appendix B].

Example: Let $X$ be a zero mean Gaussian random variable with unknown variance which belongs to the set $\left\{\sigma_{1}^{2}, \sigma_{2}^{2}\right\}$. Without the loss of generality we assume that $0<\sigma_{1}^{2}<\sigma_{2}^{2}$. The AWGN observation channel is modeled by

$$
Y=X+N, \quad N \sim \mathcal{N}\left(0, \sigma_{N}^{2}\right) .
$$

It can be seen that the compression-identification rate region $\mathcal{R}_{1}$ is given by

$$
\begin{aligned}
R_{c} & \geq \frac{1}{2} \log _{2} \frac{\sigma_{1}^{2}}{\sigma_{Y_{1}}^{2} 2^{-2 R_{i}}-\sigma_{N}^{2}} \\
0 \leq R_{i} & <\frac{1}{2} \log _{2}\left(1+\sigma_{1}^{2} / \sigma_{N}^{2}\right),
\end{aligned}
$$

where $\sigma_{Y_{1}}^{2}=\sigma_{1}^{2}+\sigma_{N}^{2}$. The achievability follows from Theorem 1 with test channels $p_{U \mid X, 1}$ and $p_{U \mid X, 2}$ such that conditioning on each state we can write

$$
X=U+N^{\prime},
$$

where $U$ and $N^{\prime}$ are independent Gaussian rvs. More specifically, the distribution of $U$ conditioning on the first state is given by $P_{U, 1}=\mathcal{N}\left(0, \sigma_{Y_{1}}^{2}\left(1-2^{-2 R_{i}}\right)\right)$. Similarly we have $P_{U, 2}=\mathcal{N}\left(0, \sigma_{Y_{2}}^{2}\left(1-2^{-2 R_{i}}\right)\right)$. The converse holds due to the entropy power inequality. The example illustrates that our scheme needs to adapt to the worst state.

\section{B. Proof of Theorem 1}

1) Achievability: Let $T_{X}$ be a classifier for $s$ and $T_{Y}$ be a classifier for $\kappa$ from Lemma 1.

Let $U$ be a random variable ${ }^{4}$ such that given $S=s$ and $X=x$ it is distributed according to the law $P_{U \mid X=x, S=s}$. Also fix a $\delta>0$.

For each $s \in \mathcal{S}$ generate $2^{n R_{c}}$ sequences $u^{n}\left(m_{s}\right) \sim P_{U \mid S=s}$, $m_{s} \in\left[1: 2^{n R_{c}}\right]$, where $P_{U \mid S=s}$ is the marginal corresponding to $P_{U \mid X, S=s}$. Hence, we have a total $|\mathcal{S}| 2^{n R_{c}}$ codeword sequences which are used to enroll all users' data.

For each $s \in \mathcal{S}$ and $\tau \in \mathcal{T}$ assume that the tuple

$$
\left(\hat{X}^{n}, \hat{Y}^{n}, \hat{U}^{n}\right) \sim\left(P_{U X \mid S=s} \times P_{Y \mid X, \tau}\right)^{\otimes n} .
$$

Denote $\mathcal{A}_{s}=\mathcal{A}_{\delta}^{n}\left(P_{X U \mid S=s}\right)$, where the latter is the weak typical set. Note that

$$
\operatorname{Pr}\left\{\left(\hat{X}^{n}, \hat{U}^{n}\right) \notin \mathcal{A}_{s} \mid S=s\right\} \rightarrow 0, \text { as } n \rightarrow \infty .
$$

Moreover, define

$$
\phi_{s, \tau}\left(x^{n}, y^{n}, u^{n}\right)=\chi_{\left\{\left(y^{n}, u^{n}\right) \notin \mathcal{A}_{\delta}^{n}\left(P_{Y U \mid \tau s}\right)\right\}},
$$

\footnotetext{
${ }^{3}$ We take the first one in the lexigraphical order if there are multiple arguments that achieve that maximum value.

${ }^{4}$ We can choose for each $s$ a different random variable $U_{s}$. Herein we abuse the notation for brevity.
}

then

$$
\begin{aligned}
\delta_{n, s \tau} & =\mathbb{E}\left[\phi_{s, \tau}\left(\hat{X}^{n}, \hat{Y}^{n}, \hat{U}^{n}\right) \mid S=s, T=\tau\right] \\
& \rightarrow 0, \text { as } n \rightarrow \infty,
\end{aligned}
$$

due to the weak law of large numbers. Define, hence,

$$
\begin{array}{r}
\mathcal{B}_{s, \tau}=\left\{\left(x^{n}, u^{n}\right) \mid \mathbb{E}\left[\phi_{s, \tau}\left(x^{n}, \hat{Y}^{n}, u^{n}\right) \mid \hat{X}^{n}=x^{n},\right.\right. \\
\left.S=s, T=\tau] \leq \delta_{n, s \tau}^{1 / 2}\right\},
\end{array}
$$

Accordingly, let $\hat{\mathcal{A}}_{s}=\mathcal{A}_{s} \cap \bigcap_{\tau} \mathcal{B}_{s, \tau}$. Note that by Markov's inequality

$$
\begin{aligned}
\operatorname{Pr}\left\{\mathcal{B}_{s, \tau}^{c} \mid S=s\right\} & \leq \frac{\mathbb{E}\left[\phi_{s, \tau}\left(\hat{X}^{n}, \hat{Y}^{n}, \hat{U}^{n}\right) \mid S=s, T=\tau\right]}{\delta_{n, s \tau}^{1 / 2}} \\
& =\delta_{n, s \tau}^{1 / 2} \rightarrow 0, \forall \tau \in \mathcal{T}
\end{aligned}
$$

so that

$$
\operatorname{Pr}\left\{\hat{\mathcal{A}}_{s} \mid S=s\right\} \rightarrow 1, \text { as } n \rightarrow \infty .
$$

Enrollment: For each user $i$, we first let $\hat{s}_{i}=T_{X}\left(x^{n}(i)\right)$. Assume that $\hat{s}_{i} \in \mathcal{S}$. Then we search for a codeword such that

$$
\left(x^{n}(i), u^{n}\left(m_{\hat{s}_{i}, i}\right)\right) \in \hat{\mathcal{A}}_{\hat{s}_{i}} .
$$

We then store the corresponding pair of $\left(\hat{s}_{i}, m_{\hat{s}_{i}, i}\right)$ as $j_{i}$ in the database. Note that when either $\hat{s}_{i}=e$ or $m_{\hat{s}_{i}, i}=e$, i.e., there does not exist a codeword such that the above condition is satisfied, the corresponding stored index is $e$.

Identification: The processing unit first assigns the observation $y^{n}$ to one of $\left|\mathcal{P}_{\mathcal{Y}}\right|$ states or $e$. We denote the corresponding state by $\kappa^{\star}=T_{Y}\left(y^{n}\right)$. It also votes for majority which state is most likely from the database using the mechanism from Lemma 2 , which is denoted by $s^{\star}$. If $\kappa^{\star}$ is not compatible with $s^{\star}$, i.e., if $\kappa^{\star}=e$ or $s^{\star}=e$ or

$$
\nexists \tau: P_{Y, \kappa^{\star}}=P_{Y \mid X, \tau} P_{X \mid S=s^{\star}},
$$

the processing center aborts the operations and declares an error. Otherwise, denote the corresponding channel index by $\hat{\tau}$. We then search for a unique $\hat{w}$ such that

$$
\left(y^{n}, u^{n}\left(m_{\hat{\hat{s}_{\hat{w}}}, \hat{w}}\right)\right) \in \mathcal{A}_{\delta}^{n}\left(P_{Y U \mid \hat{\tau} s^{\star}}\right),
$$

where $P_{Y U \mid \hat{\tau} s^{\star}}$ is the marginal of $P_{Y \mid X, \hat{\tau}} \times P_{X \mid S=s^{\star}} \times$ $P_{U \mid X, S=s^{\star}}$. An error is declared if there does not exist any such index or there is more than one.

Analysis: Let $\hat{S}_{W}, M_{\hat{S}_{W}, W}, S^{\star}$ and $\hat{T}$ be the corresponding random variables induced by the enrollment and identification processes. Without loss of generality we condition on the event $W=1, S=s$ and $T=\tau$. If $\hat{W} \neq 1$ then at least one of the event in (24) holds.

$$
\begin{aligned}
\mathcal{E}_{e s} & =\left\{S^{\star} \neq s\right\} \cup\left\{T_{X}\left(X^{n}(1)\right) \neq s\right\} \cup\left\{T_{Y}\left(Y^{n}\right) \neq \kappa\right\} \\
\mathcal{E}_{n o, s c} & =\left\{\left(Y^{n}, U^{n}\left(M_{\hat{S}_{1}, 1}\right)\right) \notin \mathcal{A}_{\delta}^{n}\left(P_{Y U \mid \hat{T} S^{\star}}\right)\right\}, \\
\mathcal{E}_{\geq 2, s c} & =\left\{\exists w^{\prime} \neq 1 \mid\left(Y^{n}, U^{n}\left(M_{\hat{S}_{w^{\prime}}, w^{\prime}}\right)\right) \in \mathcal{A}_{\delta}^{n}\left(P_{Y U \mid \hat{T} S^{\star}}\right)\right\} .
\end{aligned}
$$


The (conditional) probability of estimation error $\mathcal{E}_{e s}$ goes to zero by Lemma 1 and 2 . Additionally, it can be shown, see [11] for full proof, that if

$$
\begin{aligned}
& R_{c}>\max _{s} I(X ; U \mid S=s)+\delta \\
& R_{i}<\min _{s} I(Y ; U \mid T=\tau, S=s)-3 \delta
\end{aligned}
$$

then

$$
\begin{aligned}
& \operatorname{Pr}\left\{\mathcal{E}_{\text {es }}^{c} \cap \mathcal{E}_{n o, s c} \mid W=1, S=s, T=\tau\right\} \rightarrow 0, \\
& \operatorname{Pr}\left\{\mathcal{E}_{\text {es }}^{c} \cap \mathcal{E}_{\geq 2, s c} \mid W=1, S=s, T=\tau\right\} \rightarrow 0, \text { as } n \rightarrow \infty .
\end{aligned}
$$

This implies that the rate pair

$$
\left(\max _{s, \tau} I(X ; U \mid S=s, T=\tau), \min _{s, \tau} I(Y ; U \mid S=s, T=\tau)\right)
$$

is in $\mathcal{R}_{1}$.

2) Converse for the discrete case: Assume that the pair $\left(R_{c}, R_{i}\right)$ is achievable, i.e., for every $\delta>0$ there exists an identification scheme such that

$$
\begin{gathered}
\frac{1}{n} \log \left|\mathcal{M}_{1}\right|<R_{c}+\delta, \frac{1}{n} \log |\mathcal{W}|>R_{i}-\delta, \\
\operatorname{Pr}\{\hat{W} \neq W \mid S=s, T=\tau\}<\delta, \forall s, \tau,
\end{gathered}
$$

for all $n \geq n_{0}(\delta)$. Define

$$
U_{i}=\left(J_{W}, W, X^{i-1}(W)\right), \forall i \in[1: n] .
$$

Due to the memoryless property of the observation channel we have

$$
\begin{aligned}
& P_{Y_{i} X_{i}(W) U_{i} \mid S=s, T=\tau} \\
& =P_{Y \mid X, \tau} \times P_{X \mid S=s} \times P_{U_{i} \mid X_{i}(W), S=s}, \forall i \in[1: n] .
\end{aligned}
$$

We start bounding the compression rate. For each pair $(s, \tau) \in$ $\mathcal{S} \times \mathcal{T}$ we have

$$
\begin{gathered}
n\left(R_{c}+\delta\right) \stackrel{(a)}{\geq} H\left(J_{W} \mid W, S=s, T=\tau\right) \\
\quad \geq I\left(X^{n}(W) ; J_{W} \mid W, S=s, T=\tau\right) \\
\quad \geq \sum_{i=1}^{n} I\left(X_{i}(W) ; U_{i} \mid S=s, T=\tau\right) .
\end{gathered}
$$

(a) is valid since $\frac{1}{n} \log \left|\mathcal{M}_{1}\right|<R_{c}+\delta$ holds. Also for each pair $(s, \tau) \in \mathcal{S} \times \stackrel{n}{T}^{n}$

$$
\begin{aligned}
& n\left(R_{i}-\delta\right) \leq H(W \mid S=s, T=\tau) \\
& \stackrel{(c)}{\leq} I\left(Y^{n} ; W \mid S=s, T=\tau,\left(J_{i}\right)_{i=1}^{M}\right)+1+\delta \log |\mathcal{W}| \\
& \leq I\left(Y^{n} ; W,\left(J_{i}\right)_{i=1}^{M} \mid S=s, T=\tau\right)+1+\delta \log |\mathcal{W}| \\
& \stackrel{(d)}{=} I\left(Y^{n} ; W, J_{W} \mid S=s, T=\tau\right)+1+\delta \log |\mathcal{W}| \\
& \stackrel{(e)}{\leq} \sum_{i=1}^{n} I\left(Y_{i} ; U_{i} \mid S=s, T=\tau\right)+1+\delta \log |\mathcal{W}| .
\end{aligned}
$$

(c) holds due to Fano's inequality and since $W$ is independent of $\left(T, S,\left(J_{i}\right)_{i=1}^{M}\right) .(d)$ is valid since

$$
\left(Y^{n}, J_{W}\right)-(W, S, T)-\left(J_{i}\right)_{i=1, i \neq W}^{M}
$$

Finally $(e)$ is valid since conditioning on $T=\tau$ and $S=s$, $Y_{i}$ are independent. Let $Q$ be a uniform random variable on $[1: n]$ which is independent of everything. Define further $U=\left(U_{Q}, Q\right)$. Then we obtain, $\forall(s, \tau) \in \mathcal{S} \times \mathcal{T}$

$$
\begin{aligned}
R_{c}+\delta & \geq I\left(X_{Q}(W) ; U \mid S=s, T=\tau\right) \\
\left(R_{i}-\delta\right)(1-\delta) & \leq I\left(Y_{Q}(W) ; U \mid S=s, T=\tau\right)+\delta .
\end{aligned}
$$

Note that

$$
\begin{aligned}
& P_{Y_{Q} X_{Q}}(W) U \mid S=s, T=\tau \\
& =P_{Y \mid X, \tau} \times P_{X \mid S=s} \times P_{U \mid X_{Q}(W), S=s} .
\end{aligned}
$$

Since each conditional distribution $P_{U \mid X_{Q}}(W), S=s$ acts independently, we can upper bound the cardinality ${ }^{5}$ of $\mathcal{U}$ by $|\mathcal{X}|+|\mathcal{T}|$ by following [12, Appendix C] as each $P_{U \mid X_{Q}, S=s}$ affects $|\mathcal{T}|$ terms $H\left(Y_{Q} \mid U, S=s, T=\tau\right)$. This implies that $\left(R_{c}+\delta,\left(R_{i}-\delta\right)(1-\delta)-\delta\right) \in \overline{\mathcal{R}}_{1}$. Hence taking $\delta \rightarrow 0$ we obtain the desired conclusion.

\section{IDENTIFICATION PROBLEM: ARBITRARILY VARYING SETTINGS}

\section{A. Independent individual states}

We consider the scenario where for each user $i \in \mathcal{W}$ the state $s_{i}$ is generated from the distribution $P_{S}$. Then the corresponding data sequence is generated independently from $P_{X \mid S=s_{i}}^{\otimes n}$. The observation channel is assumed to be given as $P_{Y \mid X}$.

This scenario differs from the one given in Section II. Herein the users' data are independent from each other, whereas in the setting in Section II they are independent only given $S$. Note that in both cases, without conditioning on $S=s$ (or $S_{i}=s$ ) each user's data are not iid generated. The definition of an identification scheme is identical as before. Similarly, we have the following definition of achievability.

Definition 4. A pair $\left(R_{c}, R_{i}\right)$ is achievable if for every $\delta>$ 0 there exists a compression-identification pair of mappings $(\phi, \psi)$ such that

$$
\begin{aligned}
& \frac{1}{n} \log \left|\mathcal{M}_{1}\right|<R_{c}+\delta, \quad \frac{1}{n} \log |\mathcal{W}|>R_{i}-\delta, \\
& \sup _{\left(s_{i}\right)_{i=1}^{M} \in \mathcal{S}^{M}} \operatorname{Pr}\left\{\hat{W} \neq W \mid\left(S_{i}\right)_{i=1}^{M}=\left(s_{i}\right)_{i=1}^{M}\right\}<\delta,
\end{aligned}
$$

for all $n \geq n_{0}(\delta)$. The set of all achievable pairs is denoted by $\mathcal{R}_{3}$.

We denote also $\overline{\mathcal{R}}_{1}$ to be its restriction to the current setting, i.e., $|\mathcal{T}|=1$. The result for the current setting is summarized in the following theorem ${ }^{6}$.

Theorem 2. Given the channel $P_{Y \mid X}$ and the set of distributions $\mathcal{P}$, we have

$$
\mathcal{R}_{3}=\overline{\mathcal{R}}_{1}
$$

${ }^{5}$ The bounding procedure may result in different random variables $U_{s}$ and test channels $P_{U \mid X, S=s}$ for different $s$.

${ }^{6} \mathrm{By}$ applying the strong converse statement in [13, Theorem 4], the results of Theorem 1 and Theorem 2 could be strengthened straightforwardly in the discrete case. 
when both $X$ and $Y$ are finite. In case both $X$ and $Y$ are continuous in which the conditional density $p_{Y \mid X}$ is the channel and $\mathcal{P}$ is the set of density functions then $\mathcal{R}_{1} \subseteq \mathcal{R}_{3}$.

In the proof of Theorem 2 we use the same enrollment scheme as the one in Theorem 1 with a more careful analysis.

Compared to the setting of Theorem 1, the underlying state can be different from user to user. Hence, the processing unit can only estimate the state of the true user based on the observation sequence $y^{n}$ from the known observation channel $P_{Y \mid X}$.

\section{B. A connection to Wyner-Ahlswede-Körner network}

We establish herein a connection of the cerntain identification problem with the lossless source coding with coded side information, which sheds some light on the behavior of our settings. Recall that a code for Wyner-Ahlswede-Körner network, called a WAK-code, [9], [10] for the pair of discrete memoryless sources $\left(X^{n}, Y^{n}\right) \sim P_{X Y}^{\otimes n}$ consists of three mappings,

$$
\begin{gathered}
\phi_{1}: \mathcal{X}^{n} \rightarrow \mathcal{M}_{1}, \quad \phi_{2}: \mathcal{Y}^{n} \rightarrow \mathcal{M}_{2}, \\
\psi: \mathcal{M}_{1} \times \mathcal{M}_{2} \rightarrow \mathcal{Y}^{n} .
\end{gathered}
$$

A pair $\left(R_{1}, R_{2}\right)$ is achievable if it fulfills the following constraints for every $\delta>0$

$$
\begin{aligned}
& \frac{1}{n} \log \left|\mathcal{M}_{i}\right|<R_{k}+\delta, k=1,2, \\
\operatorname{Pr}\left\{Y^{n} \neq \hat{Y}^{n}\right\} & <\delta,
\end{aligned}
$$

for all $n \geq n_{0}(\delta)$. The set of all achievable rate pairs is denoted by $\mathcal{R}_{\text {WAK }}$. The region $\mathcal{R}_{\text {WAK }}$ is characterized by the following conditions

$$
\begin{aligned}
R_{1} & \geq I(X ; U), R_{2} \geq H(Y \mid U), \\
U & -X-Y, \quad|\mathcal{U}| \leq|\mathcal{X}|+1 .
\end{aligned}
$$

If $|\mathcal{S}|=|\mathcal{T}|=1$ then both settings in Theorem 1 and Theorem 2 collapse to the same setup. We denote the corresponding region by $\mathcal{R}_{\mathrm{ID}}$. From the trade-off characterized in Theorem 1 and the trade-off given in (36) it is immediate that $\left(R_{c}, R_{i}\right) \in$ $\mathcal{R}_{\mathrm{ID}}$ if and only if $\left(R_{c}, H(Y)-R_{i}\right) \in \mathcal{R}_{\mathrm{WAK}}$. This observation was also noticed in [14, Section III.E]. In both problems $R_{i}$ characterizes the number of confused (coded) codewords that the system can tolerate. In fact, the two networks are quite intimate which is shown by the following proposition. It states that given an arbitrarily achievable scheme for the WAKsetting we can construct a corresponding achievable code for the identification setting with the corresponding rate pair.

Proposition 1. Assume that $\left(R_{1}, R_{2}\right) \in \mathcal{R}_{\mathrm{WAK}}$, where $R_{2}<$ $H(Y)$ then there exists an identification scheme based on the corresponding WAK-code such that $\left(R_{1}, H(Y)-R_{2}\right)$ is in $\mathcal{R}_{\mathrm{ID}}$.

Consider case that in settings of Theorem 1 and Theorem 2 the observation channel is known. Let $\left\{\left(\phi_{1, s}, \phi_{2, s}, \psi_{s}\right)\right\}_{s \in \mathcal{S}}$ be an arbitrary set of mappings for the WAK-problems where the corresponding joint distributions are $\left\{P_{Y \mid X} \times P_{X \mid S=s}\right\}_{s \in \mathcal{S}}$. A code for the setting of Theorem 2 is constructed as follows. In the enrollment phase we first estimate the state $\hat{s}_{i}$ and use the corresponding mapping $\phi_{1, \hat{s}_{i}}$ to compress the data of the $i$-th user. In the identification phase the processing unit estimates the underlying state $s^{\prime}$ and uses the corresponding sets $\mathcal{B}_{m_{1}, s^{\prime}}=\left\{y^{n} \mid y^{n}=\psi_{s^{\prime}}\left(m_{1}, \phi_{2, s^{\prime}}\left(y^{n}\right)\right)\right\}$ as decision regions. Then we obtain the following upperbound for the second setting

$$
\begin{aligned}
& \operatorname{Pr}\left\{\hat{W} \neq w \mid W=w,\left(S_{i}\right)_{i=1}^{M}\right\} \\
& \leq P_{\mathrm{WAK}}\left(\text { error } \mid S=s_{w}\right)+P(\text { estimation error }) \\
& +\operatorname{Pr}\left\{\tilde{Y}^{n} \notin \mathcal{T}_{\epsilon}^{n} \mid S=s_{w}\right\}+|\mathcal{W}|\left|\mathcal{M}_{2, s}\right| 2^{-n\left(H\left(Y \mid S=s_{w}\right)-\gamma_{n}\right)} .
\end{aligned}
$$

The last term in (37) is valid since conditioning on underlying states the independence still holds. A similar upperbound can be shown for the setting in Theorem 1 specialized to the known observation channel case.

In [11] we provide an inner bound and an outer bound for the region of the setting with arbitrarily varying observation channel. In the case when compression rate is large enough, both regions coincides. While the capacity for the arbitrarily varying channel using deterministic code is either zero or equal to the capacity achieved using random code, our result does not suffer from such dichotomy.

\section{REFERENCES}

[1] F. Willems, T. Kalker, and J.-P. Linnartz, "On the capacity of a biometrical identification system," in 2003 IEEE International Symposium on Information Theory. IEEE, p. 82.

[2] E. Tuncel, "Capacity/storage tradeoff in high-dimensional identification systems," IEEE Trans. Inf. Theory, vol. 55, no. 5, pp. 2097-2106, 2009.

[3] E. Tuncel and D. Gündüz, "Identification and lossy reconstruction in noisy databases," IEEE Trans. Inf. Theory, vol. 60, no. 2, pp. 822-831, 2014.

[4] M. B. Westover and J. A. O'Sullivan, "Achievable rates for pattern recognition," IEEE Trans. Inf. Theory, vol. 54, no. 1, pp. 299-320, 2008.

[5] D. Blackwell, L. Breiman, and A. Thomasian, "The capacity of a class of channels," The Annals of Mathematical Statistics, pp. 1229-1241, 1959.

[6] - "The capacities of certain channel classes under random coding," The Annals of Mathematical Statistics, vol. 31, no. 3, pp. 558-567, 1960.

[7] R. Ahlswede, "Elimination of correlation in random codes for arbitrarily varying channels," Probability Theory and Related Fields, vol. 44, no. 2, pp. 159-175, 1978.

[8] I. Csiszar and J. Körner, Information theory: coding theorems for discrete memoryless systems. Cambridge University Press, 2011.

[9] R. Ahlswede and J. Körner, "Source coding with side information and a converse for degraded broadcast channels," IEEE Trans. Inf. Theory, vol. 21, no. 6, pp. 629-637, 1975.

[10] A. Wyner, "On source coding with side information at the decoder," IEEE Trans. Inf. Theory, vol. 21, no. 3, pp. 294-300, 1975.

[11] M. T. Vu, T. Oechtering, M. Skoglund, and H. Boche, "Uncertainty in identification systems," Manuscript in preparation.

[12] A. El Gamal and Y.-H. Kim, Network information theory. Cambridge university press, 2011.

[13] L. Zhou, V. Y. F. Tan, and M. Motani, "Exponential Strong Converse for Content Identification with Lossy Recovery," ArXiv e-prints, Feb. 2017. [Online]. Available: https://arxiv.org/abs/1702.06649

[14] C. Tian and J. Chen, "Successive refinement for hypothesis testing and lossless one-helper problem," IEEE Trans. Inf. Theory, vol. 54, no. 10, pp. 4666-4681, 2008. 